\title{
Eradication of Smallpox and Prospects for Measles Eradication: Lessons from the Brazilian Experience
}

\author{
Homma A* Possas C, Menezes R and Risi JB
}

Bio-Manguinhos, Oswaldo Cruz Foundation, Rio de Janeiro, Brazil

\begin{abstract}
There is a consensus in the scientific community that the global eradication of measles is more difficult than it was for smallpox. In the case of measles, epidemiological, social and programmatic conditions contribute to the complexity of this challenge in diverse national scenarios. In addition, the greater transmissibility of the virus requires almost $100 \%$ vaccine coverage for susceptible people. Nevertheless, success in some developing countries, such as Brazil, indicates that measles eradication is technically and operationally feasible, using existing National Vaccination Programs, adequate strategies and society mobilization. We illustrate this argument with the successful Brazilian experience in national eradication strategies, with smallpox and polio eradication and advances in measles eradication (now limited to imported cases), with the support of an adequate infra-structure for vaccine production and immunization in the main public manufacturer, Bio-Manguinhos. The evaluation of the national eradication experiences in developing countries, such as the Brazilian case, can provide, in the Decade of Vaccines (DOV), new insights and alternatives. Increasing the international support to measles eradication, which could be turning it soon into a curiosity of the past?
\end{abstract}

Keywords: Eradication; Measles; Smallpox; Brazilian immunization program; Decade of vaccines

\section{Introduction}

Remarkable progress has been made in the global immunization field in the last four decades, with eradication of smallpox, significant advances in national immunization programs in the developing nations, GAVI's introduction of new vaccines (pentavalent, rotavirus, pneumococcal) and prospects for routine immunization of HPV in the poorest countries [1].

Recently, the international debate on the Global Vaccine Action Plan for the Decade of Vaccines Collaboration, WHO [2,3], has provided new insights to accelerate and to extend vaccine introduction in the global scenario and has contributed to envisage a future where a number of goals will have been achieved within this period: polio will have been eradicated and progress will be made towards the elimination of measles, rubella and neonatal tetanus.

Nevertheless, in order to meet these goals, it will be necessary to deal with the complexity of the global context for eradication of vaccine preventable diseases, particularly in the poorest developing countries, focusing on their diverse epidemiological, social and programmatic conditions.

Measles shares several biological and epidemiological characteristics with smallpox which favor eradication: as indicated by Morgan [4], humans are the only reservoir for the virus; measles causes a visible illness; infection leads to life-long immunity; cases often occur at regular intervals enabling the targeting of interventions; measles virus has only one genetic serotype which is relatively stable over time; an effective vaccine is available and accurate laboratory identification is possible.

International and multilateral organizations such as WHO, UNICEF and PAHO and non-governmental organizations, such as GAVI, with the support of the Bill and Melinda Gates Foundation (BMGF), have certainly contributed to significant advances in the immunization area and they are already playing an important role in the measles' global eradication strategy. In order to increase immunization coverage, they have supported and contributed to a spectacular progress in the national immunization programs worldwide. In many countries they have already reached high immunization coverage with traditional and new vaccines, saving the lives of millions of children exposed to immune-preventable diseases and making it possible to reach the Millenium Development Goals.

However, measles eradication seems more complex, since it requires very high immunization coverage, almost $100 \%$ among target susceptible individuals. Moreover, it became clear that two doses are necessary to obtain measles control. To reach this goal, it would be necessary to have adequate delivery of the vaccine, qualified personnel and adequate national health services, which are not generally available in many developing countries.

We examine here, from the perspective of the global eradication, the successful experience of the National Brazilian Immunization Program and its successful eradication strategies, in the eradication of smallpox, polio and progress in measles eradication, now limited to imported cases. These strategies have been supported by the main national public manufacturer, Bio-Manguinhos, an institute of the Oswaldo Cruz Foundation, Ministry of Health.

\section{Smallpox: Success and the Eradication Debate}

The global smallpox eradication is undoubtedly a history of great success and eradication strategies for other diseases should learn from this fantastic experience. Several other international programs to

${ }^{*}$ Corresponding author: Dr. Akira Homma, MD, Chairman of Policy and Strategic Council, Bio-Manguinhos, FIOCRUZ, Rio de Janeiro, Brasil, President, Developing Countries 'Vaccine Manufacturers Network, E-mail: akira.homma@bio.fiocruz.br

Received May 02, 2012; Accepted May 23, 2012; Published May 30, 2012

Citation: Homma A, Possas C, Menezes R, Risi JB (2012) Eradication of Smallpox and Prospects for Measles Eradication: Lessons from the Brazilian Experience. $J$ Vaccines Vaccin S3:001. doi:10.4172/2157-7560.S3-001

Copyright: $\odot 2012$ Homma A, et al. This is an open-access article distributed unde the terms of the Creative Commons Attribution License, which permits unrestricted use, distribution, and reproduction in any medium, provided the original author and source are credited. 
eradicate diseases (measles, rubella, neonatal tetanus) are now in place or under consideration, in the case of measles with strong support of the International Measles Initiative created in 2001.

Globally, the debate of the need and feasibility of eradication campaigns has been intense, in the evaluations of the international strategies and efforts for smallpox and polio eradication. It is true that there are great difficulties in the implementation of eradication strategies in the poorest countries, under extreme adverse social and economic conditions.

The enormous amount of investments made in the global smallpox eradication campaign alerted the international public health community to the risks of focusing exclusively on single eradication campaigns and called the attention to the urgent need to integrate eradication efforts into well-structured national health systems, eventually supported by targeted campaigns, when necessary.

Nevertheless, the implementation of integrated health services supported by a participatory health care system is obviously still an unfinished agenda in many developing countries, and it will only be obtained after decades. For this reason, it is important to stress that this shortage in the provision of social and health services should not be an argument to delay the urgently needed eradication campaigns and strategies.

Supporters of eradication campaigns argue that they have been highly cost-effective, with significant saves for the governments, benefiting particularly developing countries. Donald Henderson, the former director of the smallpox campaign, argued that the total US\$ 313 million expenditure over ten years for the campaign (over \$1 billion today) was the best global health investment ever made [5]. Based on his estimates, the eradication of smallpox has saved the world's governments more than US\$ 1 billion annually, in reduced productivity losses and medical care costs, besides indirect savings from averted deaths and disabilities [6].

In contrast, the critics of eradication campaigns alert that the polio eradication effort has long bypassed the cost and time required by smallpox eradication. Twenty-two years into the campaign, almost \$USD 10 billion has been spent, and the presence of wild poliovirus or resurgence in several developing countries has required a re-evaluation of polio eradication strategies. These critics also argue that eradication campaigns usually require a high recipient country spending, and are often subject to aid conditionalities and unfair incentives, which may impair health systems as a whole.

In the future eradication efforts (measles, rubella, neonatal tetanus and other diseases), it will be necessary to promote the integration of the eradication campaigns vertical strategies with the national health systems horizontal strategies, approaching epidemiological, social and health services from a broad and interdisciplinary science and technology perspective. Only with this integrative approach it will be possible to provide the necessary conditions for greater efficiency and effectiveness of the epidemiological surveillance and control strategies.

\section{Background: Measles Epidemiology, Vaccine Cover- age and Strategies}

Measles meets some of the preconditions for eradication: a) transmission only from person to person, no animal reservoir; b) effective vaccine available to interrupt transmission; c) global perception of disease as a major public health problem; d) there is sufficient global interest, with political will and resources [4,7].
However, compared to smallpox, measles virus is considerably more contagious, capable of causing large outbreaks even in populations with high vaccine coverage, requiring almost $100 \%$ coverage of target susceptible populations. Moreover, below one year of age measles vaccine is less effective and it became clear that two vaccine doses are needed for measles control.

Recently, significant progress has been made in the world on vaccine coverage and measles' control. From 2000 to 2010, global measles vaccine 1 dose coverage increased from $72 \%$ to $85 \%$, with approximately 1 billion children vaccinated (JAMA, 2012). Reported measles cases decreased from 2000 to 2008, remained stable in 2009, and increased in 2010.

Nevertheless, although an effective measles vaccine has been available for around 50 years, by the end of $2010,40 \%$ of countries still had not met the incidence target of $<5$ cases per million.

\section{The PAHO's Strategy}

Due to these constraints and challenges in measles' immunization and control, PAHO formulated a successful and highly effective strategy for measles eradication, with dramatic impacts, particularly in Brazil and other Latin American countries, which consisted of:

a) Initial mass vaccination campaign, including a large age range, for example, from 9 months of age to 14 years ("catch- up") without consideration to previous measles vaccine doses or history of measles disease;

b) Maintenance of routine immunization with high and uniform vaccine coverage for children with 12 months of age or older ("keep- up");

c) Implement supplementary vaccination of those municipalities or districts with less than $95 \%$ coverage on routine immunization ("mop-up").If needed, vaccinate from door to door.

d) When the cumulative number of susceptible individuals reaches the size of a birth cohort, implement a new campaign targeting this susceptible population ("follow-up"). Typically, for vaccine coverage around $80 \%$, this involved children from 1 to 4 years of age.

The PAHO strategy was very effective, with international recognition. The last autochthonous case of measles in the Region was reported in Venezuela in 2002.

PAHO has a long standing tradition in successful immunization and eradication strategies $[8,9]$. The Americas was the first region to eradicate smallpox and the last case globally reported was in Somalia in 1977. Brazil reported its last case in 1971. The Americas was also the first region to eradicate polio, with the last autochthonous case in Peru in 1991. PAHO is now targeting to eradicate rubella.

The Brazilian National Immunization Program works in close cooperation with PAHO preserved its identity and peculiarities.

\section{The Global Market Constraints to Eradication: Avail- ability of Vaccines}

The promising global scenario in the field of vaccine development, with modern biotechnology, new vaccine strategies related to DNA recombinant technology, and other technological advances, has opened a broad range of perspectives for innovators and vaccine manufacturers. 
This scenario has motivated multinational companies to make great investments in vaccine innovation and technological development, particularly in the new vaccines of high aggregate value (recombinant hepatitis $\mathrm{B}$, conjugate heptavalent pneumococcal vaccine and HPV), with high investments in technological innovation and high profits.

Unfortunately, contrasting with this scenario, these multinational companies, in their strategy to assure high financial returns, are investing in new vaccines and giving up to produce traditional vaccines of lower prices, which are essential to public health and to the eradication strategies. This shortage has led to a vaccine supply crisis in the world market, affecting particularly the poorest developing countries.

As a consequence, public and private manufacturers in developing countries are making important efforts to overcome this crisis and the shortage on the production of traditional vaccines of lower added value, but essential for the prevention of immuno-preventable diseases affecting developing countries. In fact, these vaccines account now for more than $60 \%$ of the demand from UNICEF and PAHO Rotatory Fund/WHO [10].

Some of these efforts to overcome the current gaps in immunization are related to the development of innovative strategies and to the search of alternative technologies for vaccine administration [11]. A good example is the WHO Measles Aerosol Project. Researchers are analyzing data from the final phase III trial, in which Indian children have been given measles vaccines through a mask, in a trial of a technique that could transform vaccine delivery in remote areas. If the efficiency is as high as in previous trials, which matched needle vaccination, the aerosol method could soon be rolled out, with a lower risk of infection by the needle, lower costs, and perhaps additional benefits, such as improved vaccination effectiveness for infants.

\section{Regulatory Advances and Constraints: Harmonization and Barriers}

Concerning regulation, the national and international agencies regulating pharmaceuticals and vaccines have in the last two decades significantly increased their requirements and norms, harmonizing in most cases their procedures, in an effort to adequately meet the challenges posed by the rapid advances in technological development and production of increasingly complex biological products.

As a consequence, the small laboratories for vaccine production are disappearing, in Brazil and other developing countries, because they are not able to comply with these advanced regulatory requirements. Significant investments in maintenance, modernization and infrastructure would be necessary for them to meet these new norms and procedures, required by WHO and by the National Brazilian Agency for Health Surveillance. For the success of eradication strategies, it is important to identify international strategies to overcome this shortage, since the small manufacturers of vaccines play a crucial role in the poorest countries.

For vaccines involving research with genetically modified organisms (GMOs), there is a national legal framework requiring proposals to be submitted to the National Technical Commission on Biosafety (CTNBio), supported by a network of Local Biosafety Committees (CiBios) in research institutes and universities.

In the clinical studies, which are essential for vaccine development, proposals have to be submitted to a strict ethical evaluation by the National Committee for Ethics in Research (CONEP) supported by a large network of local ethical committee (CEPs) in research institutes and universities

The investments in vaccine innovation and technological development are a long-term and complex strategy, with implies high costs and uncertain outcome. Some stages require specific submissions and petitions, with the support of multidisciplinary teams and adequate laboratory infra-structure. This would require complex stages of validation of methodologies, equipment, installations and all other components in the development process.

\section{Populational Mobility Constraints: International Certificate of Vaccination}

The global situation at the time of the eradication of the smallpox was quite different from today. We have had in the last four decades an exponential increase in the populational mobility, aggravated by a greater global interconnectivity, resulting mainly from the international travels. As a consequence, the conditions for the transmissibility of the viruses increased dramatically. This new scenario affects even the developed countries and emerging ones, like Brazil, where immunization coverage is very high. These countries suffer now with the importation of cases from measles, polio and others viroses. Therefore, this problem has become a global problem, affecting all nations. We need to eradicate these diseases globally, for a safe and protected world. There is an urgent need, in order to confront the consequences of intense mobility, to introduce globally the requirement for international travelers, of a compulsory International Certificate of Vaccination polio, measles and rubella, following the routine adopted in some countries for the yellow fever vaccine.

\section{The Successful National Immunization Program (PNI) in Brazil}

In Brazil, vaccination coverage between 1995 and 2002 reached 95\% for DTP vaccine and $100 \%$ for polio, BCG, and MMR vaccines. Polio was eradicated in 1994, smallpox in 1971 and Brazil has registered no autochthonous measles cases since 2001. Furthermore, the country has brought diphtheria, whooping cough, and tuberculous meningitis under control. Bio-Manguinhos partnerships permitted the rapid introduction of new vaccines into the routine immunization schedule, with control of Haemophilus influenzae type b infections, and more recently, impact on rotavirus and pneumococcus infections.

The Brazilian government political and financial commitments to measles prevention and control has favored measles vaccine high and uniform coverage with 2 doses in all Brazilian regions, through routine services in the Brazilian National Health System and other Supplemental Immunization Activities in national or regional vaccine campaigns. The national strategy has been supported by a strict surveillance system for the disease and monitoring of coverage with the first and second dose of measles vaccine.

This strategy has contributed to guide interventions and to support the rapid increase of coverage, both at the national and at the local levels.

\section{The Brazilian National Immunization Program and Prospects for Measles Eradication}

The Brazilian National Immunization Program is considered one of the most complete and effective national immunization programs amongst the developing countries. It has been pioneer in the introduction of rotavirus vaccine in 2007, and in 2010 of pneumococcus 
10-valent conjugate vaccine and meningococcus $C$ conjugate vaccine. The introduction of these vaccines and the vaccination against influenza pandemic H1N1 in 2009 demonstrates the technical and operational capacity of the PNI and the Ministry of Health, including the issues concerning the logistic for immunization.

The Brazilian government immunization policy focuses on the universal access to vaccination, targeting children, adolescents, aged, Indians, providing access to most of the available vaccines in the international market. This policy resulted, besides the successful global eradication of smallpox, in the important elimination of polio, measles (now limited to imported cases) and rubella.

The Brazilian National Immunization Program is comparable to the national immunization programs from developed countries. Its rapid growth can be demonstrated by the following numbers: in 2000, its budget was R $\$ 200$ million; it had been increased to R \$ 825 million in 2009, offering 26 different types of vaccines, and now in 2012 it has been increased to US $\$ 1.3$ billion. It should be noted that the costs of H1N1 vaccine are not included in this last value, since due to its emergency nature; it received extraordinary financial resources, based on a provisory legal instrument by the federal government.

Additionally, the Brazilian immunization policy has been focused on increasing the investments in technological innovation and production, in order to stimulate the introduction of new vaccines and to strengthen the capacity of national manufacturers. This strategy, based on the sustainability of the National Immunization Program, has improved the technological qualification of national manufacturers as well as the infra-structure for innovation, development and production.

\section{Bio-Manguinhos: Brazilian Public Manufacturer}

In Brazil, Bio-Manguinhos, a FIOCRUZ's Institute (Immunobiological Technology Institute) established in 1976, has been investing strongly in the production of vaccines, kits for laboratory diagnosis and biopharmaceutical products to meet public health demands. It develops a portfolio of 35 projects with a team of 140 professionals, including 12 doctors and 50 masters. Some of these projects are still in clinical studies (phase II), such as vaccines against serogroups $\mathrm{B}$ and $\mathrm{C}$ meningococcus.

Currently the Institute is the main immunobiological provider to the Brazilian Ministry of Health, supplying $47 \%$ of the vaccines demand under the National Immunization Program (PNI). Bio-Manguinhos produces more than 60 million doses of vaccines for the National Immunization Program the United Nations Agencies. It also provides about three million kits for laboratory diagnosis per year to the Ministry of Health for the different national public health programs: the General Coordination of Public Health Laboratories, Health Surveillance Secretariat; to the National Department on Sexually Transmitted Diseases and Aids; to the National Epidemiological Surveillance Program, Health Surveillance Secretariat.

In order to address the national public health programs, BioManguinhos has about 1.000 employees and one of the largest and most modern industrial vaccine plants of Latin America: the Vaccines Technological Complex (CTV), where the Final Processing Department (DEPFI) is located, with a production of 300 million vaccine doses per year and the highest lyophilization (freeze drying) capacity of Latin America. The Institute also fulfills Good Manufacturing Practices (GMP) requirements and their products are inspected and approved by the National Institute for Quality Control, as well as by the National
Health Surveillance Agency. The issue of quality of its products has been of highest priority to Bio-Manguinhos and the Ministry of Health.

Bio-Manguinhos has also developed several international technological transfer agreements, including partnerships with other public institutes, such as the Butantan Institute, in São Paulo, Ezequiel Dias Foundation, in Minas Gerais, and other national institutes.

Since 2001, the Institute has been prequalified by the World Health Organization (WHO) to supply the yellow fever vaccine to the United Nations Agencies and to make direct exportations to other countries. Furthermore, the Institute is represented by their professionals in international organizations such as WHO, PAHO and GAVI and its former Director is now the President of the Developing Countries Vaccines Manufacturers' Network (DCVMN), a non-governmental organization connecting vaccine manufacturers from developing nations.

The national immunization policy also includes the financial support to strengthen the national research, technological and production capacity, focusing on innovation, modernization and construction of new unities and laboratories for vaccine production.

An important component of this policy is the use of the large government purchase power in the National Health System (SUS) as a strategic instrument for the technology transfer agreements. This national technological transfer strategy strengthens the national capacity and supports the production and provision of essential vaccines at a reasonable price, compatible with the governmental budget.

Innovation has become also a crucial component of this policy, in order to reduce technological dependence. The Brazilian government, aware of an important gap in this area, is strengthening the innovation capacity of the public manufacturers, stimulating public-private partnerships, increasing the investments in human resources, infrastructure and funding projects aiming new technological alternatives.

\section{Final Considerations}

It is important to seize this momentum of rapid changes in global immunization strategies and programs and to accelerate the eradication of measles, which remains an important global health problem, in spite of the availability of an effective and cheap vaccine. This global effort should be based on the successful international experience with smallpox eradication and on the PAHO's successful strategy for eradication of measles in the Americas, which in our view should be a reference for other emerging and poor countries. We understand that this PAHO strategy is the only one that would allow very high immunization coverage in these countries.

The Brazilian experience can also provide a good contribution for these countries, with the success of the National Immunization Program in the eradication of smallpox, polio and the progress in eradication of measles, indicating that this strategy is technically and operationally feasible for a developing country.

The sustainability of immunization programs is a crucial issue in many developing and remains a challenge, in spite of increasing international and national efforts.

For this reason, for the success of the measles eradication strategy, it will be necessary to increase vaccine awareness by governmental authorities and societies in these countries, with creative educational and cultural strategies focused on the importance of immunizations 
Citation: Homma A, Possas C, Menezes R, Risi JB (2012) Eradication of Smallpox and Prospects for Measles Eradication: Lessons from the Brazilian Experience. J Vaccines Vaccin S3:001. doi:10.4172/2157-7560.S3-001

Page 5 of 5

and on the need to gradually increase the national health budget dedicated to these activities.

Information should also be provided to policy makers and society on the social and moral gains of saving lives of the more vulnerable and poorest populations and on the cost-effectiveness of eradication strategies.

It will also be necessary to strengthen the capacity of the vaccine manufacturers, stimulating public-private partnerships and supporting sustainable funding initiatives and Advanced Market Commitment strategies, such as the one created by governments in developed nations and BGMF [12] to accelerate the introduction of conjugate pneumococcal vaccine.

Finally, these strategies should be supported by a global negotiation aiming to introduce urgently an International Certificate of Vaccination, compulsory for international travelers, for polio, measles and rubella, similar to the one for yellow fever required by some countries. This Certificate would contribute to restrict and control the importation of new cases of these diseases by countries which have already made an important eradication effort, such as Brazil.

These new approaches will certainly create the necessary conditions for the success of measles and other eradication strategies, in order to meet the Millenium Development Goals and to start in this century a new era of immunization, with the Decade of Vaccines as a landmark.

\section{References}

1. World Health Organization (WHO)/The United Nations Children's Fund (UNICEF)/World Bank (2009) State of the world's vaccines and immunization. $3^{\text {rd }}$ ed. Geneva: WHO.

2. World Health Organization (WHO) (2011) Draft global vaccine action plan: update. EB130/21, 15 December 2011.

3. Homma A, Leal ML, Freire MS, Couto AR (2011) Atualizacao em Vacinas Imunizacoes e Inovacao Tecnologica. Ciencia \& Saude Coletiva 16: 455-458.

4. Morgan OWC (2004) Following in the footsteps of smallpox: can we achieve the global eradication of measles? BMC International Health and Human Rights 4: 11-29.

5. Fenner F, Henderson DA, Arita I, Jesek Z, Ladnyil D (1988) Smallpox and its eradication. Geneva. WHO.

6. Birn AE (2011) Small(pox) success? Ciencia \& Saude Coletiva 16: 591-597.

7. Quadros CA (2011) The power of vaccines to eradicate diseases: smallpox, polio, measles and rubella. Anais do II Simposio Internacional de Imunobiológicos, Rio de Janeiro, 4-6 Maio.

8. Organização Pan-Americana da Saúde (Opas)/Organização Mundial da Saúde (OMS). Programa de vacinação. 2010.

9. Pan American Health Organization (PAHO)/World Health Organization (WHO (2009) Immunization newsletter: The ProVac Initiative. Washington: PAHO.

10. Milstien JB, Kaddar M (2010) The role of emerging manufacturers in access to innovative vaccines of public health importance. Vaccine 28: 2115-2121.

11. Weniger BG, Papania MJ (2008) Alternative vaccine delivery methods. In Plotkin SA, Orenstein WA, Offit PA. Vaccines. $5^{\text {th }}$ ed. Philadelphia: Saunders \& Elsevier 1357-1392.

12. Advance Market Commitment (AMC), 2010
This article was originally published in a special issue, Measles and Smallpox: Epidemiology and Immunization handled by Editor(s). Dr. Jacobson Robert M, Pediatrics College of Medicine, USA 